\title{
Popularity Aware Limited Caching for Reliable On Demand P2P Video Streaming
}

\author{
T. Suresh \\ Department of Computer Science Engineering, \\ Annamalai University, Chidambaram, Tamilnadu, \\ India, Pin: 608002
}

\author{
K. Venkatachalapathy, PhD. \\ Department of Computer Science Engineering, \\ Annamalai University, Chidambaram, Tamilnadu, \\ India, Pin: 608002
}

\begin{abstract}
In nowadays network group, the Peer-To-Peer (P2P) network is exploring as a good environment for resource sharing over the Internet. Compared with traditional file sharing workloads, continuous streaming of multimedia content provokes a significant amount of today's internet traffic. Streaming media has various real-time constraints such as insufficient memory, high bandwidth utilization for largescale media objects, and lack of cooperation between proxies and their clients. Under these circumstances, it is a challenging task to achieve efficient content delivery under the increased availability of continuous-media streaming. In this paper, PALC (Popularity Aware Limited caching) is proposed to achieve efficient, reliable and scalable P2P-VoD system. It is an attractive approach to minimize the network traffic incurred in video streaming through cooperative caching. In this approach, P2P network is constructed in the form of a two-tier architecture, where the upper tier is composed of proxy overlay with high connectivity super peers. It serves as a structured DHT node to maintenance the detached hash table for prominent information retrieval. On the contrary, in lower tier, less connectivity peers are loosely coupled to the corresponding super peers in the form of unstructured network. We proposed an efficient hybrid proxy caching system in order to reduce the network/ server load while improving the end user perceived video quality. It also proposes an efficient Popularity Aware Limited Caching (PALC) algorithm that cooperatively utilizes the memory space of the proxy servers and clients to achieve efficient and cost effective P2P video streaming. The effectiveness of the proposed approach is evaluated under various network and end system configurations. The experimental results demonstrate that the proposed approach incurs minimum Internet traffic and low cost of transmission as compared to the proxy-based caching approach with limited storage space.
\end{abstract}

\section{General Terms}

Peer to Peer Networks, Video Streaming, Proxy Caching

\section{Keywords}

Video-on-demand (VoD), PALC (Popularity Aware Limited caching), Hybrid proxy caching, Scalability, Media Streaming

\section{INTRODUCTION}

The Video on Demand (VOD) system attains the significant position in interactive media entertainment, due to the rapid growth of the Internet. It permits users to choose and watch the desired video over a network with the engagement of VOD system. In VoD systems, video content is segmented as a stream of content that allows the user to view the video in real time. The user can select the desired video and it is retrieved as quickly as possible in the client playback device. The main disadvantage of video streaming is that users have to spend a large amount of storage resource, and downloading time in order to download the entire media content into their local disk in the playback device. The most popular networked media service in the Internet is the online video streaming with asynchronous playback requests [1]. Therefore, in recent years, a broad spectrum of applications such as distance learning and NetTV have earned considerable attention in many practical deployments. But, it is a difficult task to deploy a reliable and scalable on-demand media streaming service with high quality and low cost over the Internet under limited bandwidth resources, limited server capacity, large size of media objects, and the unpredictable Internet environment.

In the research of video streaming over the Internet [2], three remarkable solutions are available to support the efficient streaming. i) Content Delivery Network (CDN) is evolved to perform video streaming that replicates media content among the servers across the Internet. It provides an efficient data transport with high user perceived quality and less access latency. In the CDN, desired video content may be available in one of the neighbor peers due to the large number of server deployment. It is highly appreciable for efficiency but not cost-effective. ii) In the proxy assisted video streaming, proxy server effectively caches the media content among the proxy servers in the overlay network. It is cost-effective, but scalability is not much noticeable due to limited storage capacity and bandwidth availability of centralized servers. iii) The third approach is distributed client-based P2P overlay networks for media content delivery where each peer distributively caches the media content along with the proxy server in order to reduce the excessive workload of proxy servers with limited resources. This approach is cost effective and also achieves scalability, but does not meet the required quality of service. It is difficult to achieve the heterogeneity available among the large number of peers in terms of CPU, storage, and bandwidth resources [3] [4].

In order to improve the performance of video streaming [5], proxies are deployed close to the clients that can cache frequently used data to reduce server loads. Therefore, video streaming is performed with low latency and reduced network traffic. The proxy caching provides significant performance improvement in video streaming with asynchronous demands and highly localized access interests. Due to the high availability of caching resources, media objects are retrieved in high data rates with long playback durations. A lot of existing approaches relies on the proxy caching that can achieve efficient streaming for small size video content. In the case of large video content, caching a complete media objects is not practically feasible in the case of limited proxy cache space. However, in order to handle the large size media objects, segment-based proxy caching strategies are proposed. Instead of caching the entire media content, the segment based approach partially caches the segments of media objects. The segment-based proxy caching technique provides 
efficient streaming with high scalability, but the quality of service is still not satisfactory in the client side due to the following reasons i) The small size of the reference locality of multimedia objects leads to a cache space problem in media proxy systems. ii) The single point failure may occur in proxy based caching system iii) It is highly vulnerable to overload. iv) The number of client is limited due to the highly demanded dedicated reservation of proxy bandwidth for continuous streaming [6] [7]. The problems occur in client based caching are described as follows i) First, the more searching time is taken to predict the availability of demanded media among the peers in the network because lack of coordination among the peers. Therefore, each peer caches and replaces media content independently. It leads to a highly dynamic environment. ii) Due to the transient and dynamic nature of peers, service availability varies dynamically even though the data is available. iii) Due to the high collaborative nature among the multi-peers, it is difficult to maintain the Quality of Services (QoS) under highly dynamic and bursty media streaming environment.

In order to overcome the above limitations in both approaches, a new distributive approach is proposed that combines the advantages of proxy and client based caching. Proxy caches the video content already retrieved by other client in the network to reduce the communication cost instead of retrieving it from remote servers. Since memory capacity of proxy server is limited, it cannot store the large number of video content. Therefore, in this approach, client peers cache the retrieved video content in its own cache. It leads to a distributive caching where the video content is cached distributively among the proxy server and client peers in the network. In order to achieve global sharing with high scalability, media objects are cached in both the client and proxy servers. An efficient and optimal Popularity Aware Limited Cache (PALC) allocation algorithm is proposed where proxies and clients distributively caches the video segments according to the video content popularity. The transmission cost is significantly reduced and able to adapt for heterogeneous computation and storage constraints of proxies and clients. In this paper, constructed P2P network can effectively coordinate the high cooperative communication between the proxy server and client peers in the network.

\subsection{Contributions}

The main contribution of this paper is

* In this system, a two-tier P2P network is selforganized to achieve high coordination between the proxy server and $\mathrm{P} 2 \mathrm{P}$ clients. This leads to acquire high scalability where system can effectively manage and perform on demand video streaming.

* An optimal cache allocation scheme is proposed to achieve effective cooperation among the multiple proxy servers with client caching.

* Popularity aware limited caching is a heuristic algorithm used to obtain efficient cache replacement policy, and it dynamically adjusts the distribution of media data cached in the system.

* In order to utilize the efficient data locality, segment-based replacement policies are performed among the proxy servers and client peers in the system.

* The main objective of this paper is that the proxy maintains the popular media segments for global sharing for a long while. In order to maintain the unpopular segments, a small space is left for each peer in the network.
* The overall hit ratio is improved due to the effective and adaptive maintenance of popular and unpopular segments.

\subsection{Paper organization}

The rest of the paper is organized as follows: In Section 2, we review the related work. Section 3 elucidates the proposed system architecture. We derive efficient algorithms for optimal cache allocation and popularity aware limited caching. The performance of the proposed system is extensively evaluated in Section 4. Finally, we concluded the work in Section 5.

\section{RELATED WORK}

This section analyzes the existing works on $\mathrm{P} 2 \mathrm{P}$ video on demand streaming, an investigation is carried out on various approaches such as Proxy assisted selective catching [8], SURFNet [9], Segment-based caching proxy (SProxy) [10], Dynamic segment-based Caching Algorithm (DECA) [11] and optimal proxy cache allocation algorithm [12].

Proxy assisted selective catching is the hybrid combination of both proxy-assisted catching and controlled multicast [8]. Video objects are classified in to the popular (hot) and unpopular objects (cold) depending upon the user access patterns. According to the nature of video objects, a suitable multicast scheme is deployed. If the user is interested to broadcast the popular video objects (hot) then the proxy assisted catching is used. In unpopular media content, proxy assisted controlled multicast is used that relies on client-pull technique to broadcast the video objects efficiently. This approach deploys a specific periodic broadcasting scheme called as Greedy Disk-conserving Broadcast (GDB) which is a "server-push" media content delivery technique used to broadcast periodically through the dedicated channels. In this scheme, video objects are partitioned into segments, and it is periodically broadcasted through dedicated channels to achieve continuous video streaming in the playback device. This hybrid approach outperforms either proxy-assisted catching or controlled multicast separately in terms of service latency and bandwidth consumption.

SURFNet achieves user interactivity that provides high reliability and fast content discovery mechanism content in $\mathrm{P} 2 \mathrm{P}$ video-on-demand (VoD) system [9]. Here, a two tier P2P network structure is constructed where the upper tier is built in the form of a structured network with high connectivity super peers, and lower tier is constructed as a unstructured network for efficient information exchange in loosely manner. The well connected super peer overlay is gossip based unstructured network to provide efficient video streaming at high transmission rate and low latency. Videos are segmented into two types of segments such as superchunk, and chunk to support different search granularities. Due to the construction of the AVL tree using the stable peers, data availability is increased among the super-peers in the overlay. Hence, peers connected to the stable peers are grouped together into a holder-chain in the form of a chunk tree. The key enhancement in this approach is i) Structured overlay is integrated with distributed gossip protocol to provide efficient search and retrieval. ii) The AVL tree is efficiently constructed with the super-peers in the overlay where the depth of the tree is determined from the number of super chunks available to construct the AVL tree.

A segment-based caching proxy (SProxy) is proposed [10] to support concurrent multimedia streaming to the limited users 
with low access latency and to manage the limited resource constraints. This system consists of four main components such as the streaming engine, segmentation-enabled cache engine, the Local Content Scheduler (LCS) module and a high speed disk. Streaming engine acts as a user interface and a segmentation- enabled cache engine act as a server interface for the video content. LCS module coordinates the functionality of both the streaming engine and the cache engine. Due to the availability of high speed availability of disk space, a fast video content delivery is supported. The SProxy is efficiently designed and implemented to enable the existing Internet infrastructure with high content distribution under low access latency. This scheme provides an efficient mean to deliver the multimedia content in content server with high scalability and efficiency. The segment-based scheme intrinsically diminishes the startup latency and also achieves continuous streaming even under various network conditions.

In work [11], an efficient Dynamic segment-based Caching Algorithm (DECA) is proposed to provide high satisfaction for the user. It also acquires the high trade off between the initial delay and the deviation of the starting point. This scheme is extended through innovative features such as segment-prefix structure, video popularity and early start concepts in existing caching schemes. The main enhancement in DECA approach is that the segment size of the video content is dynamically varying according to the updated video popularity. Here, the size of the segment is determined from the estimated weighted user satisfaction, the initial delay and the early start time. In order to achieve high average user satisfaction, the DECA approach attains the fair tradeoff between the initial delay and an early start. This caching scheme supports high user interactivity to enable efficient cache replacement policy, when compared to other existing segment-based caching approaches in video streaming.

\section{OVERVIEW OF POPULARITY AWARE PARTIAL CACHING}

In this proposed approach, a two tier P2P network is constructed with the set of proxy servers and group of client peers in the network. A peer wants to download a video, forwards the query request into its home proxy that generates the streaming schedule through processing the client request. In the approach, proxy server maintains the cached fragments and also maintains the index of the client peer which caches the streamed segments of the video. Therefore, the segmented media and its corresponding indices are decoupled in the proxy servers. The index of a video segment is effectively derived from the location of peers, a cached media segment and the retrieval information of the corresponding segment. It is mainly used for replacement operations. An efficient and optimal cache allocation is developed to cache the media content among the peers in the network. Then, popularity aware limited caching is proposed to achieve efficient cache replacement according to the popularity of the video content.

The proposed approach is composed of three main components such as Streaming server, Proxy server and the client peers. Streaming server consists of media objects in its video repository, and then the proxy is the bootstrap server which acts as an interface between the media server and the client server. Proxy server fetches the requested media content available in the media server, and then it caches the fetched content in the form of small segments which are obtained through the partitioning the entire fetched media content from the media server. Client peers can generate the request and receiving the media streaming service.

\subsection{P2P network construction}

In this work, P2P network is constructed in the form of a two tier network. Here, the upper tier is composed of a set of proxies which are unified together as the structured $\mathrm{P} 2 \mathrm{P}$ overlay. Whereas in the lower tier, set of client peers are closely connected with the high connectivity proxy in order to provide an efficient video content delivery with high scalability under large video size. In the constructed P2P network, Proxy server in the upper tier takes responsibility to perform content searching and then client peers in the lower tier responsible for chunk level information exchange. Proxy overlay is constructed through the logical connection between a set of proxy servers using direct or indirect links. A group of client peers in the same ISP domain are connected to any of the proxy server in the overlay network. Thus, the group of client peers closely connected to its home proxy performs efficient and reliable online video streaming [13].

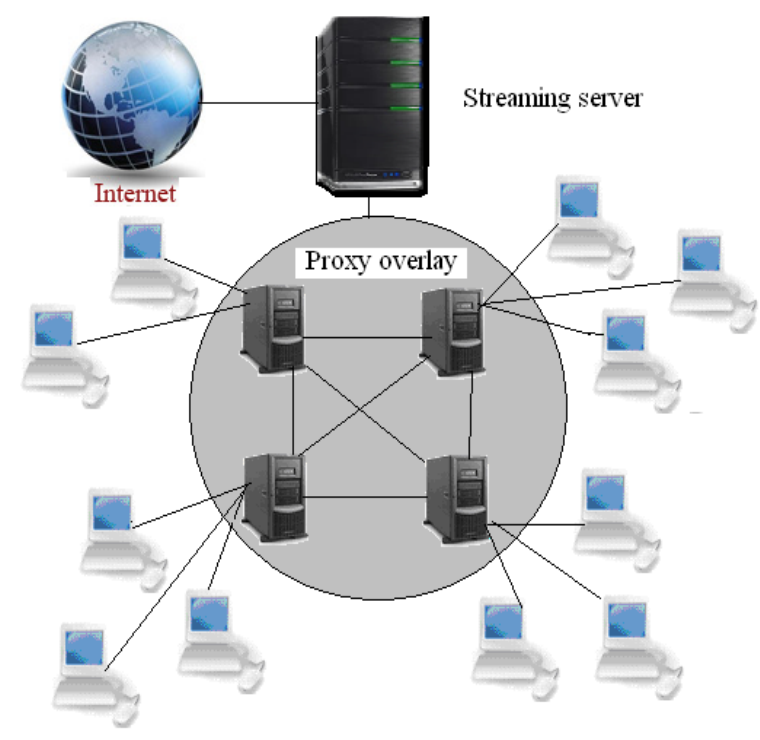

Fig 1Two tier P2P network Construction

In Fig1, the P2P network is effectively constructed with well-connected peers where each peer can play four mutually independent roles as follows: i) First, a peer can act as a client for requesting media content from the proxy peers in the overlay network. ii) Peer also performs the role of streaming server to provide the requested media from the peers in the network. iii) Each peer also acts as a storage device while caching the segmented media content in order shares the cached data with other peers in the system. iv) A peer is also an index server in order to locate the media content. It maintains a group of derived indices of media segments in the system. Proxy servers in this system are self-organized into a structured proxy overlay where each proxy server maintains the distributed hash table (DHT). It is used to map the identifier of the media segment to the index of the media segment.

\subsection{Video segmentation}

The proposed scheme partitions the video contents into segments with fine granularity to achieve low communication overhead. Therefore, the finely segmented video segment is 
more efficient for cache utilization. The proposed scheme partitions the video object into two types of interleaved fragments such as cached fragments (Cfrag) and streamed fragments (Sfrag). The cached fragments of video are its prefix, and the proxy peer takes responsibility to cache the prefix of the video. Whereas the client peer caches the streamed fragments (Sfrag) of the video which is the prefixof-suffix of the video. Here, the proxy server caches the initial part of the video because it is frequently retrieved by most of the users in the network. Therefore, it can improve the initial playback delay and also facilitate to achieve efficient multicast delivery under high peer dynamics.

\subsection{Proxy overlay maintenance}

The media segments and indices of client peers are efficiently decouples in the proxy server with the help of the distributed hash table. Each proxy server in P2P overlay has DHT maintains the (key; value) pairs to map the index information of its connected client peers. Here, the key represents the identifier which is a Globally Unique IDentifier (GUID) hashed from the address of the media object and its corresponding value denotes the index of the media segment. A new client peer joins to the proxy server through assigning the key space zone. It also maintains the segment indices to map the video content in the zone. In order to achieve P2P routing with efficient locating and data management, five important operations such as publish, unpublish, request, update, and notify are performed using the information in the DHT.

\subsection{Announcing of video media segments}

A peer can advertise the information about the cached media segment through the function called Advertise (Id; Position) to announce the information to all other peers in the network, where id is the hashed identifier of the segmented media content and position location of the peer that caches the segment copy. Correspondingly, the unadvertised (Id; position) operation unpublishes the copy of the media segment stored in the location. The advertise operation is performed to route its location to the target peer that maintains the segmented index. The unadvertised operation is performed to remove the peer from the location list of the segment index. Proxy server performs effective segmentation process and also derives the index for the segmented video content. Index server takes complete responsibility to maintain the cache consistency between the media segments and corresponding indices. A peer announces a segment as soon as it caches the full segment and then the unadvertised operation is called to delete the segment indices. A peer wants to join in the system means then it publishes the segment indices. On the contrary, in order to leave from the system, peer unpublishes all the cached segment indices in its cache [14].

\subsection{Cache Lookup Retrieval Algorithm}

In order to locate the requested segment, the proposed approach performs the four sequential steps as follows

i) STEP1: The requesting peer searches in its own disk space and local cache. If the local search fails, then request (Id; URL) operation is forwarded to the target peer. The requested peer generates the requests for the desired video segment with its corresponding identifier and the designated location. ii) STEP2: Peer routes the request to the target peer using the designated URL in the requested operation. It also maintains the key space zone in order to identify the mapped identifier (id) of the segmented media content. If the target peer finds the segment index, two cases are possible

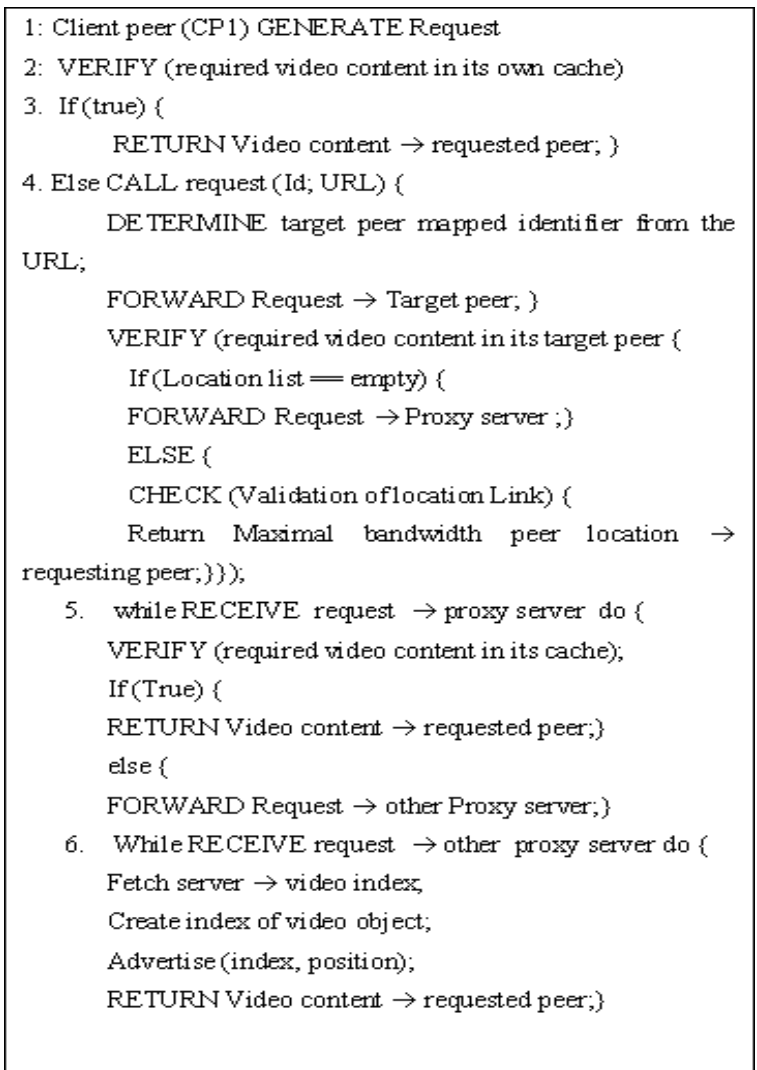

- The location list is empty: If the location list of the target peer is empty, then it forwards a request to the corresponding proxy server. Proxy server maintains the indices of segmented media segments in its connected client peers.

- The location list is not empty: If the location list of the target peer is accompanied with the location, then the target peer validates the location link and chooses the best link which is most suitable for effective media content delivery with maximum bandwidth. It returns the selected peer location to the requesting peer. If the corresponding index is not existing, it resembles that video object is requested for the first time. Then the request is forwarded to its corresponding proxy server.

iii) STEP3: The target peer forwards a request to the proxy when the location list of the target is empty. Proxy server fetches the requested object from the streaming server. After that index is created for the fetched media segment and then publishes it over the network. In order to get the required media content from the proxy server, target peers redirect the proxy location to the requesting peer. The proxy server processes the request through searching whether the requested video segment is available in its cached fragments. If it is available, then the request is forwarded into corresponding client peers which caches the streamed segments.

iv) STEP4: Otherwise request is forwarded to other proxy servers in the overlay network. Then, the other proxy peers process the request provides the requested data to the 
requesting peer. Here, client can buffer the next media segment when the current segment is played back. If a peer intentionally wants to leave from the network before the current video streaming process terminates, then it must push the remaining segment to the requesting peer before leaving from the network.

\subsection{Popularity-Aware Limited caching}

In this paper, Popularity-Aware Partial caching algorithm is proposed to perform the effective cache management based on the popularity and utility values of the video content. Here, popularity of the video segment isiii) determined from the number of times accessed by the peers in the network over the time. Obviously, a more efficient cache replacement algorithm should remove the media segment with lower popularity rather than the most popular video with high popularity. In order to achieve the finest access granularity, proxy server derives the index from the cached data item independently. Therefore, the popularity of every cached stream under infinite interval is computed, not the entire video. Once serving the requested peer, the update (ID, access information) is called to update the access information of the target peer, which maintains the segment's index of the segmented video content. By using notify (Peerset, id, value) operation, index server notifies the segment popularity and utility value to the other peers in the network. The peer set represents a set of peers in the location list of the segment index, and its corresponding identifier. The value represents the popularity or utility value of the segment designated by id. The popularity of the video segment is determined from the Zipf-like distribution.

According to the popularity value, video segments are ranked in the descending order. The popularity of the kth media segment pi is determined as follows

$$
\text { Popularity }=\frac{\mathrm{D}}{\mathrm{k}^{\Theta}}
$$

Where $\mathrm{D}=\sum 1 / \mathrm{k}^{\theta} \mathrm{k} \in\{1 \ldots \mathrm{m}\}, \mathrm{m}$ is the number of video segments, and $\theta$ is a numerical constant. Due to the efficient distributed indexing and caching schemes in the proposed scheme that can achieve efficient scalability and reliable media content delivery, than that of traditional proxy caching systems.

\section{PERFORMANCE EVALUATION}

In this section, a PeerSim is used to simulate the proposed popularity aware limited caching and illustrates the simulation setup and performance evaluation metrics to evaluate the effectiveness of the proposed scheme. Network topology is constructed with 10,000 peers in two stage power law distribution and each peer possesses 24 neighbor peers. Experimental investigation is carried out to evaluate the effectiveness of mechanism introduced in the proposed scheme. It evaluates the effect of network traffic, success rate, response time and cache hit ratio of cached video content.

\section{Average network traffic}

Average network traffic $=\Sigma \mathrm{Ti}$

$$
\Sigma \text { Qi }
$$

Where $\mathrm{Ti}$ is the total amount of traffic generated by queries Qi is the total number of queries generated by peer $i$. ii) Success Rate: The success rate of P2P searching is explicitly relying on how query processing is efficiently performed.

$$
\text { Success Rate }=\frac{\sum \mathrm{Si}}{\Sigma \mathrm{Qi}}
$$

Where $\mathrm{Si}$ is the number of successful searches done by peer $\mathrm{i}$.

Qi is the number of queries generated by peer $\mathrm{i}$.

Cache Hit Ratio: It is defined as the ratio of requested queries satisfied through cached information rather than broadcasting of query message.

$$
\text { Cache Hit Ratio }=\frac{\Sigma \mathrm{Ci}}{\Sigma \mathrm{Qi}}
$$

Where $\mathrm{Ci}$ is the number of successful searches routed through cache

Qi is the number of queries generated by peer $\mathrm{i}$.

The performance evaluation of the proposed system is carried out with two key performance metrics such as searching efficiency and cache hit ratio. The experimental result is portrayed in Fig 2.

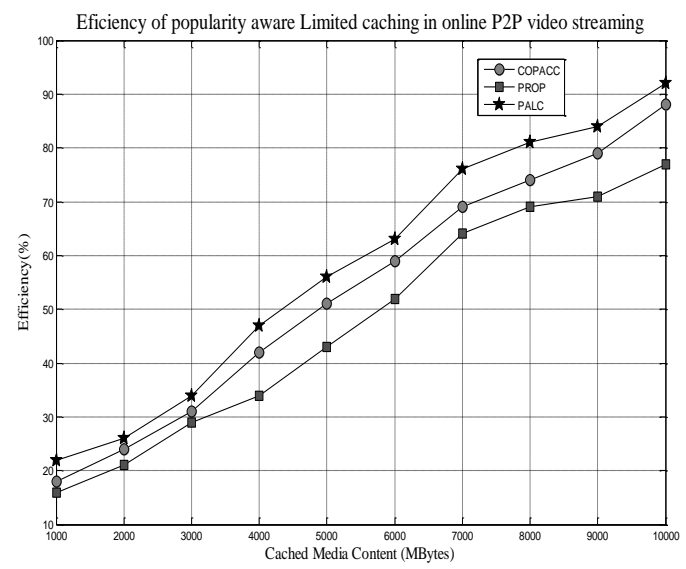

Fig 2: Efficiency of PALC on demand P2P video Streaming

The Fig 2 shows the comparison of the proposed PALC caching scheme with the existing segment based proxy assisted caching scheme. It is clearly depicted that proposed approach achieves high efficiency than existing approaches, due to the optimal cache allocation and popularity aware cache replacement policies. It is highly resilient to network failure because the high cooperation exists between all peers in the network. The proposed PALC achieves high efficiency according to the increased number of cached content among the proxy server and client peers in the network. Therefore, optimal cache allocation with popularity aware limited caching improves the efficiency of the proposed scheme in accordance to the increased number of cached content. 


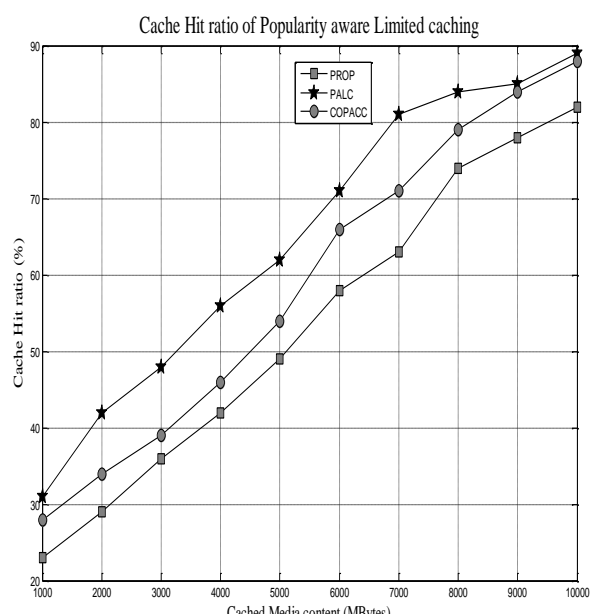

Fig 3: Cache Hit Ratio of PALC on demand video Streaming

The Fig 3 shows the comparison performed based on the Cache Hit Ratio (CHR) between the proposed PALC caching scheme and existing segment based proxy assisted caching schemes. It is clearly depicted that the proposed approach achieves high cache hit ratio than existing approaches, because the proposed scheme distributively caches the video content among the proxy servers and peers in the network. Due to the popularity aware caching leads to the effective maintenance of the cached content with cache replacement policies. Therefore, the increasing number cached media content to satisfy the most of the client request due to the optimal cache allocation and popularity aware cache replacement policies.

\subsection{Summary of Results}

The proposed approach performs effective $\mathrm{P} 2 \mathrm{P}$ video streaming with reduced transmission cost is due to the following for two reasons i) First, the proxy servers in proxy overlay has high connectivity to all peers in the network. Therefore, it can able to maintain access information of segments and also can manage the high workload with the help of index server. ii) A more efficient cache replacement algorithm is proposed that provides high preference to store the most popular video with high popularity rather than the media segment with less popularity.

* Efficiently constructed two-tier P2P network is coupled with optimal popularity aware limited caching, that provides reliable segmented video streaming for large size video objects.

* Under optimal popularity aware limited caching, a relatively small proxy cache $(10 \%-20 \%$ of the video repository) can provide efficient video streaming with less transmission cost.

* The proposed approach substantially achieves minimum transmission cost for $\mathrm{P} 2 \mathrm{P}$ caching, even in the presence of high arrival rates. It also achieves high scalability.

* Carefully designed P2P network coupled with optimal cooperative proxy and client caching can produce significant cost savings over using unicast delivery, even when the underlying network offers only unicast service.

\section{CONCLUSION}

The aim of the proposed work is to reduce the transmission cost of P2P video streaming even for asynchronous requests from the client peers. The performance of the on demand P2P video streaming in terms of scalability and reliability is improved through the efficient deployment of proxy assisted client caching system. Due to the effective construction of two-tier P2P network paved the way to attain effective cooperation between the proxy server and client peers in the network. It leads to achieve video playback quality with low latency and reduced network traffic. Completely caching the whole media content can quickly occupy the limited proxy cache space. Therefore, a scalable segment based P2P media delivering system called Popularity Aware Limited Caching is proposed that organizes the proxy servers and its clients in the same intranet into a P2P system. It achieves more robust, high cost-effective and high-quality on-demand video streaming. Experimental results demonstrate that the proposed popularity aware limited caching (PALC) approach achieves low transmission cost as compared to existing PROP and COPACC schemes with limited storage space. Furthermore, it scales well to larger networks, and the transmission cost gets reduced when more proxies and clients cooperate with each other.

\section{REFERENCES}

[1] Yong Liu, Yang Guo, Chao Liang, "A survey on peer-topeer video streaming systems", International Journal on Peer-to-Peer Networks and Applications, Vol 1, No 1, pp 18- 28, 2008.

[2] Dapeng Wu, Yiwei Thomas Hou, Wenwu Zhu, Ya-Qin Zhang, Jon M. Peha, "Streaming Video over the Internet: Approaches and Directions", "IEEE Transactions on Circuits and systems for video technology, Vol 11, No 3 , pp 282-300, 2001.

[3] Jiangchuan Liu, "Streaming Media Caching", http://citeseerx.ist.psu.edu/viewdoc/summary?doi=10.1.1 $.61 .3430,2006$

[4] Bommaiah, K. Guo, M. Hofmann, and S. Paul, "Design and Implementation of a Caching System for Streaming Media over the Internet," Proc. IEEE Real Time Technology and Applications Symp., May 2000.

[5] M. Chesire, A. Wolman, G. Voelker, and H. Levy, "Measurement and Analysis of a Streaming Media Workload," Proc. Third USENIX Symp. Internet Technologies and Systems, Mar. 2001.

[6] Z. Miao and A. Ortega, "Proxy caching for efficient video services over the internet," Proceedings of the International workshop on packet Video Workshop (PV’99), New York, Apr. 1999.

[7] R. Rejaie, M. Handley, H. Yu, and D. Estrin, "Proxy caching mechanism for multimedia playback streams in the internet," in Proceedings of the international workshop on Web Caching Workshop, San Diego, CA, Mar. 1999.

[8] Lixin Gao, Zhi-Li Zhang, and Don Towsley, "ProxyAssisted Techniques for Delivering Continuous Multimedia Streams", IEEE/ACM Transactions on Networking, Volume 11, No 6, pp 884-894, 2003.

[9] Danqi Wang and Chai Kiat Yeo, "Superchunk-Based Efficient Search in P2P-VoD System", IEEE 
Transactions on Multimedia, Vol 13, No 2, pp 376-387, 2011.

[10] Susie Wee, Xiaodong Zhang, "SProxy: A Caching Infrastructure to Support Internet Streaming", IEEE Transactions on Multimedia, Vol 9, No 5, pp 1062-1072, 2007.

[11] Wei Tu, Eckehard Steinbach, M. Muhammad, and Xiaoling Li, "Proxy Caching for Video-on-Demand Using Flexible Starting Point Selection", IEEE Transactions on Multimedia, Vol 11, No 4, pp 716-729, 2009.

[12] Bing Wang, Subhabrata Sen, Micah Adler, and Don Towsley, "Optimal Proxy Cache Allocation for Efficient Streaming Media Distribution", IEEE Transactions on Multimedia, Vol 6, No 2, pp 366-374, 2004.

[13] Alan T.S. Ip, Jiangchuan Liu, and John Chi-Shing Lui, "COPACC: An Architecture of Cooperative Proxy-Client Caching System for On-Demand Media Streaming", IEEE Transactions on Parallel and Distributed Systems, Vol 18, NO. 1, pp 70-83, 2007.

[14] Lei Guo, Student Member, Computer Society, Songqing Chen and Xiaodong Zhang, "Design and Evaluation of a Scalable and Reliable P2P Assisted Proxy for On-
Demand Streaming Media Delivery", IEEE Transactions on Knowledge and Data Engineering, VOL. 18, NO. 5, pp 669-682, 2006.

\section{AUTHORS PROFILE}

T.Suresh, working as a Assistant Professor in Department of Computer Science and Engineering, Annamalai University, Chidambaram. He has received his B.E in CSE from Shanmugha College of Engineering, Thanjavur in 1997 and M.B.A from Madurai Kamaraj University, Madurai in 2005 and M.E in CSE from Annamalai University, Chidambaram in 2007 and pursuing Ph.D in the field of Networking from Annamalai University, Chidambaram. He has teaching experience of 9 years.

Dr.K.Venkatachalapathy, working as a Professor in Department of Computer Science and Engineering, Annamalai University, Chidambaram, TamilNadu. He has received his M.C.A from Pondicherry University, Pondicherry, in 1990 and Ph.D in the field of Image Processing, from Annamalai University, Chidambaram, in 2008. He has teaching experience of 21 years. He has published 6 International journals and 8 National journals. 